\title{
Robust Self-Contained Pedestrian Navigation by Fusing the IMU and Compass Measurements via UFIR Filtering
}

\author{
Meng Hou $\mathbb{D}^{D},{ }^{1}$ Yuan $\mathrm{Xu}^{2}$, and Xiao Liu ${ }^{1}$ \\ ${ }^{1}$ School of Electrical Engineering and Automation, Qilu University of Technology, Jinan 250353, China \\ ${ }^{2}$ The School of Electrical Engineering, University of Jinan, Jinan 250022, China \\ Correspondence should be addressed to Meng Hou; houmeng@qlu.edu.cn
}

Received 31 January 2018; Revised 17 March 2018; Accepted 26 March 2018; Published 8 May 2018

Academic Editor: Choon Ki Ahn

Copyright (c) 2018 Meng Hou et al. This is an open access article distributed under the Creative Commons Attribution License, which permits unrestricted use, distribution, and reproduction in any medium, provided the original work is properly cited.

\begin{abstract}
In order to overcome the poor observability of yaw measurement for foot-mounted inertial measurement unit (IMU), an integrated $\mathrm{IMU}+$ Compass scheme for self-contained pedestrian navigation is presented. In this mode, the compass measurement is used to provide the accurate yaw to improve the accuracy of the attitude transformation matrix for the foot-mounted IMU solution. And then, when the person is in a stance phase during walk, a unbiased finite impulse response (UFIR) filter based on the self-contained pedestrian navigation scheme is investigated, which just needs the state vector size $M^{U}$ and the filtering horizon size $N^{U}$, while ignoring the noise statistics compared with the Kalman filter (KF). Finally, a real test has been done to verify the performance of the proposed self-contained pedestrian navigation using the IMU and compass measurements via UFIR filter. The test results show that the proposed filter has robust performance compared with the KF.
\end{abstract}

\section{Introduction}

Indoor pedestrian navigation (PN), which provides the position and the heading information of the target person in indoor environment, has received a topic $[1,2]$.

In order to provide the accurate position information of the person in indoor environment, many approaches have been proposed. For example, in $[3,4]$, the radio frequency identification (RFID)-based technologies have been proposed provide object self-localization. Yang and Shao and $\mathrm{Ma}$ et al. proposed in $[5,6]$ an autonomous positioning system operating on $\mathrm{WiFi}$, which is also able to achieve the indoor localization in indoor environment. However, it should be pointed out that although the RFID- and WiFibased methods mentioned above are able to provide the position information in indoor environment, the accuracy of such approaches is on meter-level. In order to improve the positioning accuracy, ultrasonic-based approaches are proposed in [7]. However, it should be pointed that although the ultrasonic-based approaches are on centimeter-level, it is easy to be outage. Meanwhile, the ultra wideband (UWB) technology [8] is employed in some approaches. For example, a location detection and tracking of moving targets by a 2D IR-UWB radar system is presented in [9]. It should be emphasized that the methods mentioned above have to employ extra infrastructures; moreover, the sampling time of these methods is larger $[1,10]$.

In order to overcome the disadvantages of the methods mentioned above, the inertial navigation systems (INS) have been employed for providing the human position in global positioning system- (GPS-) denied areas. One of the famous examples is the navigation shoe proposed in [11], which employs the foot-mounted inertial measurement unit (IMU) to correct the error drift of the INS solution. Based on this model, there are many improving approaches. For example, in [12-14], the magnetic sensor is used to correct the positioning error of the foot-mounted IMU. On the other hand, there are also many approaches for the signal processing in INS. For example, the analysis for microelectromechanical system (MEMS) gyroscope within wide-temperature range is shown in [15].

Based on the INS, the Kalman filter (KF) and its improving methods are widely used to correct the INS 
solution error [16, 17]. However, despite great progress in the development of the KF approach, the recursive KF-based algorithms demonstrate good performance mostly when the noise statistics are known exactly, and the model perfectly matches the process; otherwise, the KF-based estimators often demonstrate poor performance [18-20]. In order to provide the robust estimation, the unbiased finite impulse response (UFIR) filter has been proposed in [21-23]. Then, there are some improving UFIR filters proposed to improve the performance [24-27]. Compared with the KF-based filters, UFIR filters just need the state vector size and the filtering horizon size, while ignoring the noise statistics $[25$, $28,29]$.

In this paper, we propose a self-contained pedestrian navigation by fusing the IMU and compass measurements via UFIR filtering. To the self-contained pedestrian navigation scheme, the compass measurement is used to provide the accurate yaw to improve the accuracy of the attitude transformation matrix for the foot-mounted IMU solution. Moreover, we investigate the UFIR filter based on the selfcontained pedestrian navigation scheme, which just needs the state vector size $M^{U}$ and the filtering horizon size $N^{U}$, while ignoring the noise statistics. Finally, a real test has been done to verify the performance of the proposed selfcontained pedestrian navigation using the IMU and compass measurements via UFIR filter. The test results show that the proposed filter has robust performance compared with the KF. The remaining part of this paper is organized as follows. Section 2 designs the scheme of the self-contained pedestrian navigation using the IMU and compass measurements. Section 3 presents the UFIR algorithms for the self-contained pedestrian navigation. Testing and results are discussed in Section 4. Finally, Section 5 gives the conclusions.

\section{The Self-Contained Pedestrian Navigation Using the IMU and Compass Measurements}

In this section, the scheme of self-contained pedestrian navigation using the IMU and compass measurements will be designed. Then, the state and measurement equations based on the scheme which we designed will be investigated.

\subsection{The Scheme of Self-Contained Pedestrian Navigation Using} the IMU and Compass Measurements. In this subsection, we will introduce the scheme of self-contained pedestrian navigation using the IMU and compass measurements. The architecture of the self-contained pedestrian navigation employing the recent IMU and compass measurements is shown in Figure 1. In this work, we employ inertial measurement unit (IMU) and compass measurements for the indoor self-contained pedestrian navigation. The IMU is fixed on the shoe, and the compass is fixed on the shoulder. In this paper, the IMU is used to provide the acceleration $a$, angular velocity $w$, pitch, and the roll, which are used to compute the position and posture of the target person. Compared with the IMU fixed on the shoe, thigh, shank, and waist, the IMU fixed on

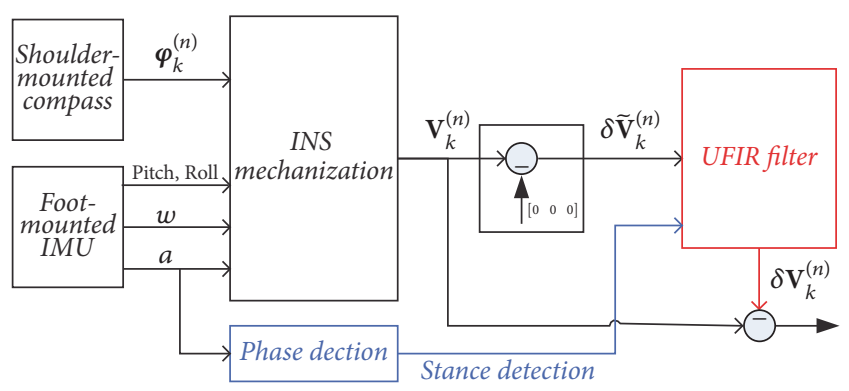

FIGURE 1: The architecture of the self-contained pedestrian navigation using the IMU and compass measurements.

the shoulder has the best performance for yaw measurement. Thus, we employ shoulder-mounted IMU to provide the yaw measurement for the solution, which can improve the accuracy of the attitude transformation matrix for the footmounted IMU solution. In theory, the true velocity of the pedestrian shoes should be zero when the shoe is on the floor (so called stance phase). Thus, the velocity measured from the foot-mounted IMU will be the error measurement of the velocity in the stance phase. And when the person is in a stance phase, the unbiased finite impulse response (UFIR) filter works; it employs the measurement of the velocity error derived from the foot-mounted IMU to estimate the INS position error; then, the INS solution is corrected by the error estimation.

Meanwhile, the body frame (so called b-frame) and the navigation frame (so called $n$-frame (East-North-Up, ENU)) used in this paper are also shown in this figure. Compared with the outdoor navigation, the area of the indoor self-contained pedestrian navigation is very small; thus, the earth's rotation is not considered in this paper.

\subsection{The State and Measurement Equations for the UFIR Filter.} In this subsection, the state and measurement equations for the UFIR filter will be designed. Based on the self-contained pedestrian navigation scheme proposed in Section 2.1, a UFIR filter with a 15-element vector will be introduced in this paper. The state equation of the UFIR filter used in this paper at time step $t t$ is listed as

$$
\begin{aligned}
\underbrace{\left[\begin{array}{c}
\phi_{t t}^{(n)} \\
\delta \mathbf{V}_{t t}^{(n)} \\
\nabla_{t t}^{(n)} \\
\varepsilon_{t t}^{(n)}
\end{array}\right]}_{\mathbf{X}_{t t}}=\underbrace{\left[\begin{array}{cccc}
\mathbf{I}_{3 \times 3} & \mathbf{0}_{3 \times 3} & \mathbf{0}_{3 \times 3} & \mathbf{I}_{3 \times 3} T \\
S\left(\mathbf{f}_{t t}^{(n)}\right) T & \mathbf{I}_{3 \times 3} & \mathbf{I}_{3 \times 3} T & \mathbf{0}_{3 \times 3} \\
\mathbf{0}_{3 \times 3} & \mathbf{0}_{3 \times 3} & \mathbf{I}_{3 \times 3} & \mathbf{0}_{3 \times 3} \\
\mathbf{0}_{3 \times 3} & \mathbf{0}_{3 \times 3} & \mathbf{0}_{3 \times 3} & \mathbf{I}_{3 \times 3}
\end{array}\right]}_{\mathbf{A}_{t t}} \underbrace{\left[\begin{array}{c}
\phi_{t t-1}^{(n)} \\
\delta \mathbf{V}_{t t-1}^{(n)} \\
\nabla_{t t-1}^{(n)} \\
\varepsilon_{t t-1}^{(n)}
\end{array}\right]}_{\mathbf{X}_{t t-1}} \\
+\omega_{t t-1},
\end{aligned}
$$

where $\left[\phi_{t t}^{(n)}, \delta \mathbf{V}_{t t}^{(n)}\right]^{T}$ denotes attitude and velocity error vectors in $\mathrm{n}$-frame at time step $t t$ and $\left[\nabla_{t t}^{(b)}, \varepsilon_{t t}^{(b)}\right]^{T}$ denotes the biases for accelerometers and gyroscopes in b-frame at time 


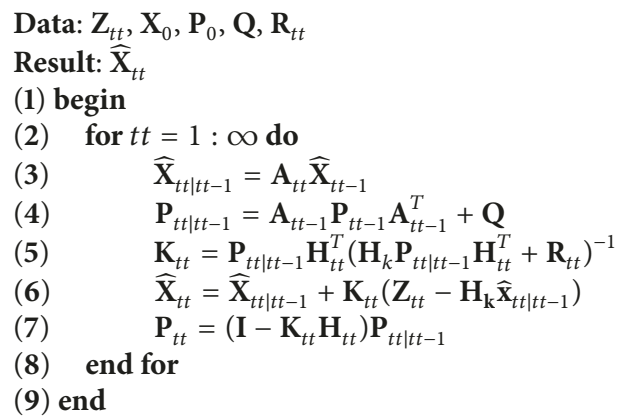

Algorithm 1: Kalman filter algorithm for the self-contained scheme.

step $t$, respectively. All these 5 components mentioned above have 3 elements each:

$$
S\left(\mathbf{f}_{t t}^{(n)}\right)=\left[\begin{array}{ccc}
0 & a_{D t t}^{(n)} & -a_{N t t}^{(n)} \\
-a_{D t t}^{(n)} & 0 & a_{E t t}^{(n)} \\
a_{N t t}^{(n)} & -a_{E t t}^{(n)} & 0
\end{array}\right]
$$

here, $\left[\begin{array}{lll}a_{E t t}^{(n)} & a_{N t t}^{(n)} & a_{D t t}^{(n)}\end{array}\right]$ is the acceleration in n-frame (EastNorth-Up, ENU) at time step $t t$; $T$ denotes the sampling time; $\omega_{t t}$ is a system noise at time step $t t$ with the covariance $\mathbf{Q}_{t t}$.

The measurement equation is listed in

$$
\underbrace{\left[\begin{array}{l}
\delta \widetilde{\mathbf{V}}_{t t}^{(n)}
\end{array}\right]}_{\mathbf{Z}_{t t}}=\underbrace{\left[\begin{array}{llll}
\mathbf{0}_{3 \times 3} & \mathbf{I}_{3 \times 3} & \mathbf{0}_{3 \times 3} & \mathbf{0}_{3 \times 3}
\end{array}\right]}_{\mathbf{H}_{t t}} \underbrace{\left[\begin{array}{c}
\phi_{t t}^{(n)} \\
\delta \mathbf{V}_{t t}^{(n)} \\
\nabla_{t t}^{(n)} \\
\varepsilon_{t t}^{(n)}
\end{array}\right]}_{\mathbf{X}_{t t}}+\eta_{t t},
$$

where $\delta \widetilde{\mathbf{V}}_{t t}^{(n)}$ is the observations for the velocity error in $\mathrm{n}$-frame at time step $t t ; \eta_{t t}$ is a measurement noise with covariance is $\mathbf{R}_{t t}$.

\section{UFIR Algorithms for the Self-Contained Pedestrian Navigation}

In this section, the KF and the UFIR filter for the indoor self-contained pedestrian navigation based on the scheme proposed in Section 2.1 will be discussed.

3.1. KF Algorithm. As one of the most used data fusion algorithm, the Kalman filter (KF) and its improving methods have been widely used in many fields [30-34]. And its pseudo code is listed as Algorithm 1. It should be pointed out that the Kalman filter and its improving methods need the accurate model description and noise description, especially the accurate $\mathbf{Q}$ and $\mathbf{R}_{t t}$, to maintain the performance. However, it is not easy to get the information mentioned above in someplace.

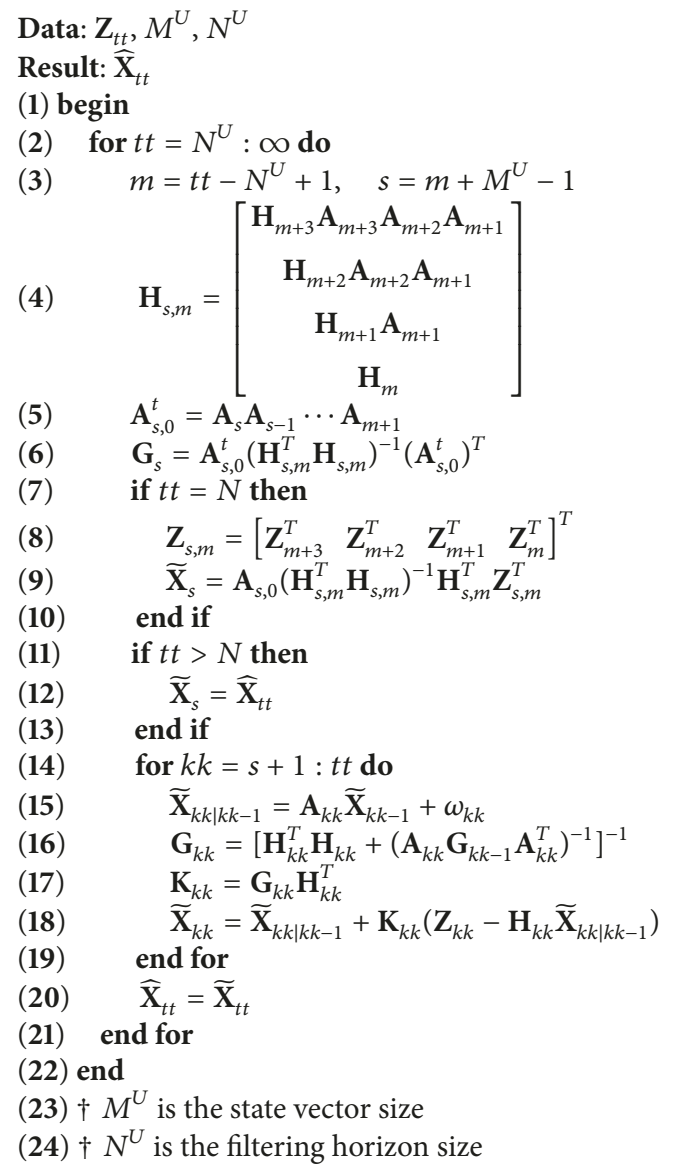

Algorithm 2: UFIR filter algorithm for the self-contained scheme.

3.2. UFIR Filtering Algorithm. Compared with KF, the UFIR filter does not need the accurate $\mathbf{Q}$ and $\mathbf{R}_{k}$ to keep its accuracy $[26,35]$. Based on the self-contained scheme proposed in Section 2.1, the UFIR filtering algorithm is listed in Algorithm 2. Thus, we can say that the UFIR filter is more robust than the KF filter.

\section{Test and Discussion}

In this paper, we employ a real indoor test to verify the performance of the proposed self-contained pedestrian navigation using the IMU and compass measurements via UFIR filter. The real test was done in the Machine Building of the University of Jinan, Jinan, China. In this section, the real test will be designed and we will investigate the corresponding results. Firstly, the real test will be designed. Then, the performances of the KF and UFIR filter will be compared.

4.1. Setting. The test platform used in the real test consists of one 9 degree of freedom (DOF) IMU, one compass, one computer, and one encoder. The 9-DOF IMU is fixed on the shoe; it employs ADXL203, ADXRS620, and HMC5983 as accelerometer, gyroscope, and magnetometer, respectively, 
TABLE 1: The parameters for the tests.

\begin{tabular}{lccccc}
\hline & $\mathbf{X}_{0}$ & $\mathbf{P}_{0}$ & $\mathbf{Q}$ & $\mathbf{R}$ & $N^{U}$ \\
\hline Group 1 & $\mathbf{0}_{12 \times 1}$ & $\mathbf{I}_{12 \times 12}$ & $\mathbf{I}_{12 \times 12}$ & $\mathbf{I}_{3 \times 3}$ & 13 \\
Group 2 & $\mathbf{0}_{12 \times 1}$ & $\mathbf{I}_{12 \times 12}$ & $10^{-2} \mathbf{I}_{12 \times 12}$ & $\mathbf{I}_{3 \times 3}$ & 13 \\
\hline
\end{tabular}

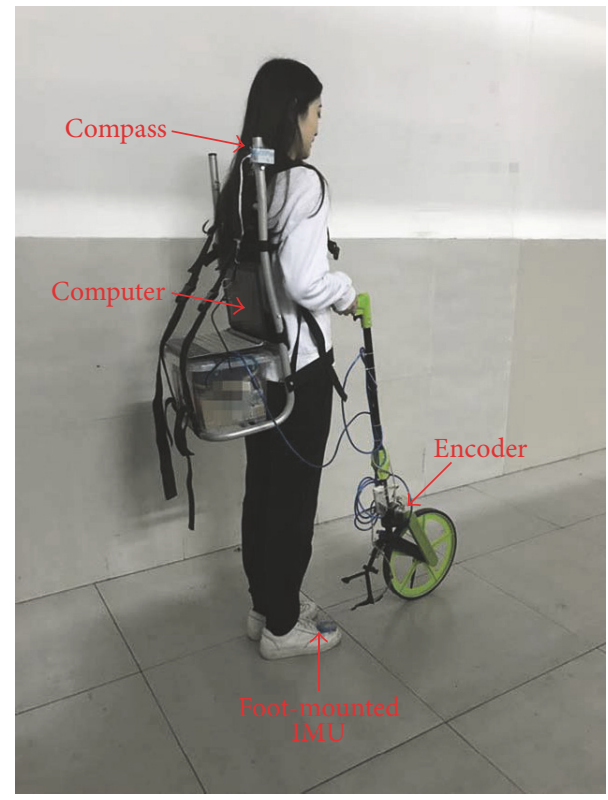

Figure 2: The prototype of the test platform.

which is able to provide the human position. Then, one HMC5983-based compass which is fixed on the shoulder is used to provide more accurate yaw measurement, which helps improve the accuracy of the yaw for the foot-mounted IMU. The computer is used to collect the sensor data. The encoder used in the real test is able to provide the reference velocity of the person. The sampling time $T$ used in (1) is $0.03 \mathrm{~s}$. From Algorithm 2, we can see that the performance of the UFIR filter is just need $M^{U}$ and $N^{U}$. From (1), we can get that $M^{U}=12$ and we employ $N^{U}=13$ in this paper. The prototype of the test platform is shown in Figure 2.

4.2. The Performance of the UFIR Filter. The position error of the UFIR filter for self-contained pedestrian navigation using the IMU and compass measurements will be discussed in this section. In this subsection, we employ two groups of parameters which listed in Table 1.

(1) INS Position Errors-Group 1. Trajectories measured by INS + ZUPT + KF and INS + ZUPT + UFIR for the first group of possible parameters (Group 1) are shown in Figures 3 and 4. From the figures, firstly, we can see that the proposed selfcontained pedestrian navigation scheme is able to provide the person position without any auxiliary equipment. Secondly, we can see that the trajectories estimated by the KF and the UFIR filter are similar.

The absolute average position errors by the KF and UFIR filter in Test 1 (Group 1) are listed in Table 2. From the table,

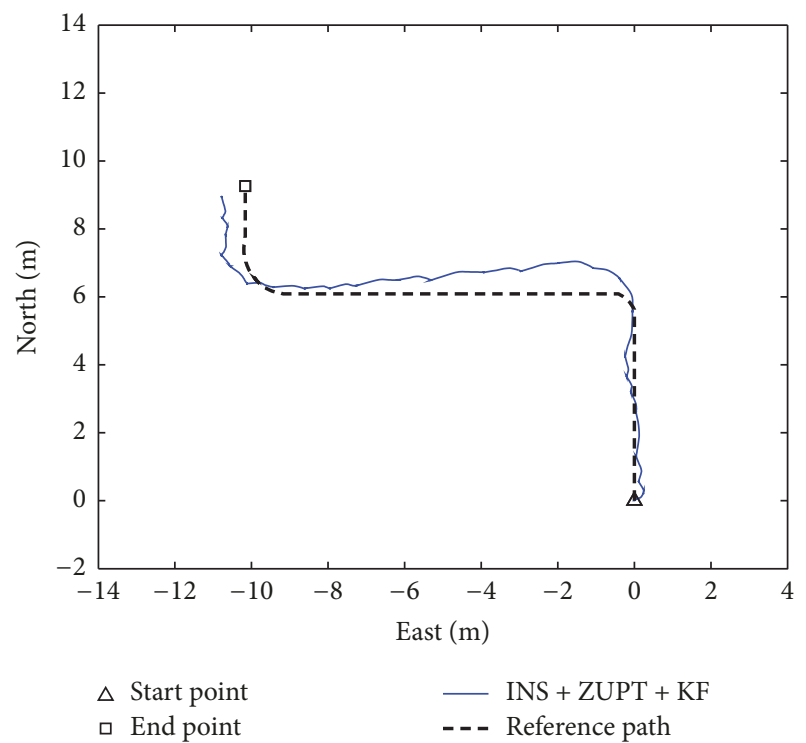

FIgURE 3: Trajectory measured by INS + ZUPT + KF for the first group of possible parameters (Group 1).

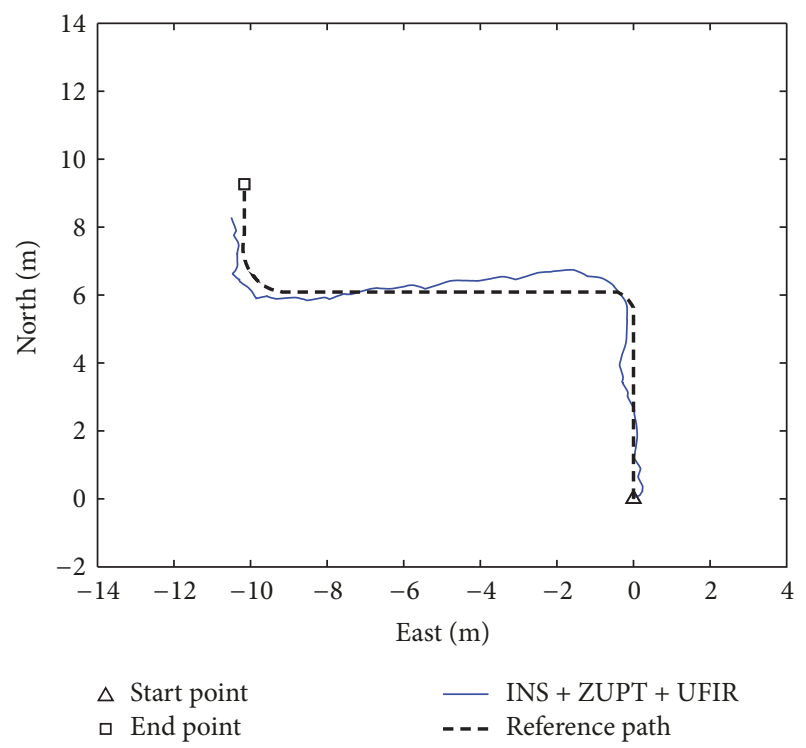

FIGURE 4: Trajectory measured by INS + ZUPT + UFIR for the first group of possible parameters (Group 1).

we can see that the position errors of the KF and UFIR filter are similar. Thus, we can see that the performances of KF and UFIR filter are similar in Test 1 (Group 1).

(2) INS Position Errors-Group 2. We now repeat the experiment for the second group of possible parameters (Group 


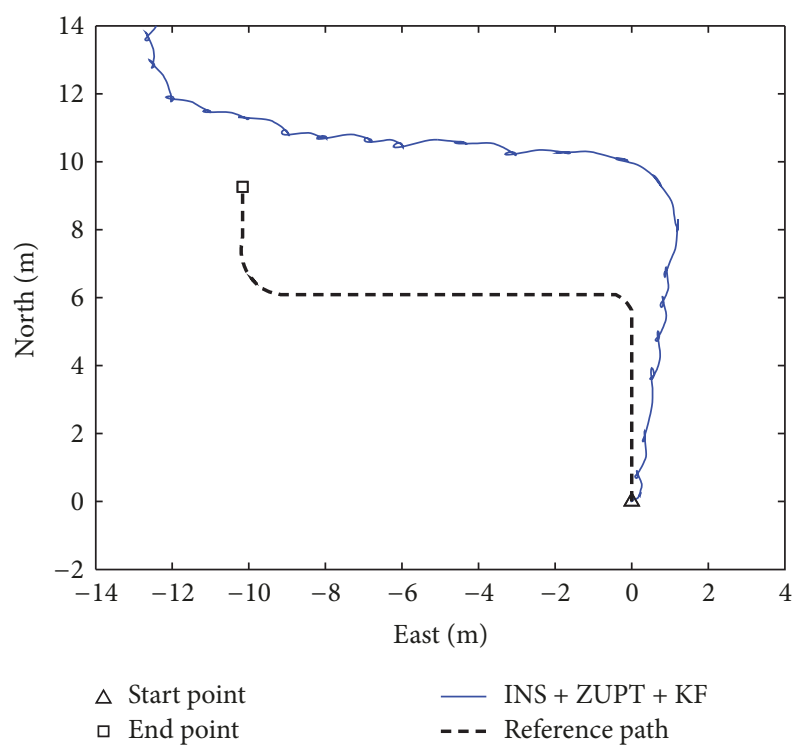

FIGURE 5: Trajectory measured by INS + ZUPT + KF for the second group of possible parameters (Group 2).

TABLE 2: Absolute average position errors by different filters in Test 1 (Group 1).

\begin{tabular}{lcc}
\hline \multirow{2}{*}{ Model } & \multicolumn{2}{c}{ Absolute average position errors [m] } \\
& East direction & North direction \\
\hline KF & 0.25 & 0.29 \\
UFIR & 0.22 & 0.31 \\
\hline
\end{tabular}

TABLE 3: Absolute average position errors by different filters in Test 2 (Group 2).

\begin{tabular}{|c|c|c|}
\hline \multirow{2}{*}{ Model } & \multicolumn{2}{|c|}{ Absolute average position errors [m] } \\
\hline & East direction & North direction \\
\hline $\mathrm{KF}$ & 0.88 & 2.79 \\
\hline UFIR & 0.22 & 0.31 \\
\hline
\end{tabular}

2). The trajectories measured from UWB only model using least square algorithm and the INS/UWB loosely coupled integrated model using the KF and UFIR filter for the second group of possible parameters (Group 2) are shown in Figures 5 and 6 . And the absolute average position errors by the KF and UFIR filter in Test 2 (Group 2) are listed in Table 3. From the figures, we can see easily that the KF has been diverged, while the performance of the UFIR filter is still good.

\section{Conclusion}

In this paper, a self-contained pedestrian navigation by fusing the IMU and compass measurements via UFIR filtering and both the implement and test are proposed. To the self-contained pedestrian navigation scheme, the compass measurement is used to provide the accurate yaw to improve the accuracy of the attitude transformation matrix for the foot-mounted IMU solution. Moreover, we investigate the

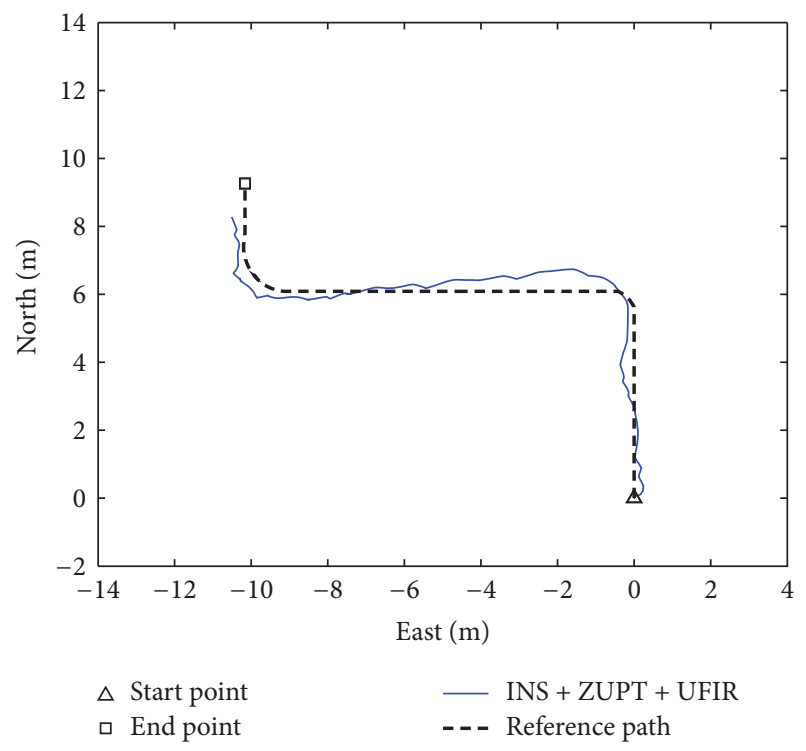

FIGURE 6: Trajectory measured by INS + ZUPT + UFIR for the second group of possible parameters (Group 2).

UFIR filter based on the self-contained pedestrian navigation scheme, which just needs the state vector size $M^{U}$ and the filtering horizon size $N^{U}$, while ignoring the noise statistics. Finally, a real test has been done to verify the performance of the proposed self-contained pedestrian navigation using the IMU and compass measurements via UFIR filter. The test results show that the proposed filter has robust performance compared with the KF.

\section{Conflicts of Interest}

The authors declare no conflicts of interest.

\section{Acknowledgments}

This work was supported in part by the China Postdoctoral Science Foundation (no. 2017M622204) and the Doctoral Foundation of the University of Jinan (no. XBS1503).

\section{References}

[1] Y. Xu, X. Chen, J. Cheng, Q. Zhao, and Y. Wang, "Improving tightly-coupled model for indoor pedestrian navigation using foot-mounted IMU and UWB measurements," in Proceedings of the 2016 IEEE International Instrumentation and Measurement Technology Conference, I2MTC 2016, Taiwan, May 2016.

[2] Y. Zhuang, H. Lan, Y. Li, and N. El-Sheimy, "PDR/INS/WiFi integration based on handheld devices for indoor pedestrian navigation," Micromachines, vol. 6, no. 6, pp. 793-812, 2015.

[3] A. Aguilar-Garcia, S. Fortes, E. Colin, and R. Barco, "Enhancing RFID indoor localization with cellular technologies," EURASIP Journal on Wireless Communications and Networking, vol. 2015, no. 1, article no. 219, 2015.

[4] J. J. Pomarico-Franquiz and Y. S. Shmaliy, "Accurate selflocalization in rfid tag information grids using fir filtering," 
IEEE Transactions on Industrial Informatics, vol. 10, no. 2, pp. 1317-1326, 2014.

[5] C. Yang and H.-R. Shao, "WiFi-based indoor positioning," IEEE Communications Magazine, vol. 53, no. 3, pp. 150-157, 2015.

[6] R. Ma, Q. Guo, C. Hu, and J. Xue, "An improved WiFi indoor positioning algorithm by weighted fusion," Sensors, vol. 15, no. 9, pp. 21824-21843, 2015.

[7] X. Chen, Y. Xu, Q. Li, J. Tang, and C. Shen, "Improving ultrasonic-based seamless navigation for indoor mobile robots utilizing EKF and LS-SVM," Measurement, vol. 92, pp. 243-251, 2016.

[8] Y. Xu, Y. S. Shmaliy, Y. Li, and X. Chen, "UWB-Based Indoor Human Localization with Time-Delayed Data Using EFIR Filtering," IEEE Access, vol. 5, pp. 16676-16683, 2017.

[9] V.-H. Nguyen and J.-Y. Pyun, "Location detection and tracking of moving targets by a 2D IR-UWB radar system," Sensors, vol. 15, no. 3, pp. 6740-6762, 2015.

[10] Y. Xu, X. Chen, and Y. Wang, “Two-mode navigation method for low-cost inertial measurement unit-based indoor pedestrian navigation," Simulation, vol. 92, no. 9, pp. 839-848, 2016.

[11] E. Foxlin, "Pedestrian tracking with shoe-mounted inertial sensors," IEEE Computer Graphics and Applications, vol. 25, no. 6, pp. 38-46, 2005.

[12] A. R. Jimenez, F. Seco, J. C. Prieto, and J. Guevara, "Indoor Pedestrian navigation using an INS/EKF framework for yaw drift reduction and a foot-mounted IMU," in Proceedings of the 2010 7th Workshop on Positioning, Navigation and Communication, WPNC'10, pp. 135-143, Germany, March 2010.

[13] Z.-Q. Zhang and X. Meng, "Use of an inertial/magnetic sensor module for pedestrian tracking during normal walking," IEEE Transactions on Instrumentation and Measurement, vol. 64, no. 3, pp. 766-783, 2015.

[14] Y. Xu, X. Chen, Q. Li, and J. Tang, "Indoor pedestrian navigation using two low-cost IMU-based framework," Journal of Chinese Inertial Technology, vol. 23, no. 6, pp. 714-717, 2015.

[15] H. Cao, H. Li, X. Shao et al., "Sensing mode coupling analysis for dual-mass MEMS gyroscope and bandwidth expansion within wide-temperature range," Mechanical Systems and Signal Processing, vol. 98, pp. 448-464, 2018.

[16] Y. Xu and X. Chen, "Online cubature Kalman filter RauchTung-Striebel smoothing for indoor inertial navigation system/ultrawideband integrated pedestrian navigation," Proceedings of the Institution of Mechanical Engineers, Part I: Journal of Systems and Control Engineering, vol. 232, no. 4, pp. 390-398, 2017.

[17] S. Zhao, Y. S. Shmaliy, P. Shi, and C. K. Ahn, "Fusion Kalman/ UFIR Filter for State Estimation With Uncertain Parameters and Noise Statistics," IEEE Transactions on Industrial Electronics, vol. 64, no. 4, pp. 3075-3083, 2017.

[18] X. Qing, H. R. Karimi, Y. Niu, and X. Wang, "Decentralized unscented Kalman filter based on a consensus algorithm for multi-area dynamic state estimation in power systems," International Journal of Electrical Power \& Energy Systems, vol. 65, pp. 26-33, 2015.

[19] H. R. Karimi, N. A. Duffie, and S. Dashkovskiy, "Local capacity ho control for production networks of autonomous work systems with time-varying delays," IEEE Transactions on Automation Science and Engineering, vol. 7, no. 4, pp. 849-857, 2010.

[20] Y. Wei, J. Qiu, H. R. Karimi, and M. Wang, "Filtering design for two-dimensional Markovian jump systems with state-delays and deficient mode information," Information Sciences, vol. 269, pp. 316-331, 2014.

[21] Y. Xu, C. K. Ahn, Y. S. Shmaliy, X. Chen, and Y. Li, "Adaptive robust INS/UWB-integrated human tracking using UFIR filter bank," Measurement, vol. 123, pp. 1-7, 2018.

[22] Y. S. Shmaliy, S. Zhao, and C. K. Ahn, "Unbiased finite impulse response filtering: an iterative alternative to Kalman filtering ignoring noise and initial conditions," IEEE Control Systems Magazine, vol. 37, no. 5, pp. 70-89, 2017.

[23] Y. S. Shmaliy, S. Khan, and S. Zhao, "Ultimate iterative UFIR filtering algorithm," Measurement, vol. 92, pp. 236-242, 2016.

[24] C. K. Ahn and Y. S. Shmaliy, "New receding horizon fir estimator for blind smart sensing of velocity via position measurements," IEEE Transactions on Circuits and Systems II: Express Briefs, vol. 65, no. 1, pp. 135-139, 2018.

[25] Y. Xu, H. R. Karimi, Y. Li, F. Zhou, and L. Bu, "Real-time accurate pedestrian tracking using extended finite impulse response filter bank for tightly coupling recent inertial navigation system and ultra-wideband measurements," Proceedings of the Institution of Mechanical Engineers, Part I: Journal of Systems and Control Engineering, vol. 232, no. 4, pp. 464-472, 2017.

[26] C. K. Ahn, Y. S. Shmaliy, and S. Zhao, "A New Unbiased FIR Filter with Improved Robustness Based on Frobenius Norm with Exponential Weight," IEEE Transactions on Circuits and Systems II: Express Briefs, 2017.

[27] S. Zhao, Y. S. Shmaliy, C. K. Ahn, and P. Shi, "Real-Time Optimal State Estimation of Multi-DOF Industrial Systems Using FIR Filtering," IEEE Transactions on Industrial Informatics, vol. 13, no. 3, pp. 967-975, 2017.

[28] H. R. Karimi and H. Gao, "Mixed H2 / Ho output-feedback control of second-order neutral systems with time-varying state and input delays," ISA Transactions ${ }^{\circledR}$, vol. 47, no. 3, pp. 311-324, 2008.

[29] H. R. Karimi, P. J. Maralani, B. Lohmann, and B. Moshiri, "ho control of parameter-dependent state-delayed systems using polynomial parameter-dependent quadratic functions," International Journal of Control, vol. 78, no. 4, pp. 254-263, 2005.

[30] S. Eftekhar Azam, E. Chatzi, and C. Papadimitriou, "A dual Kalman filter approach for state estimation via output-only acceleration measurements," Mechanical Systems and Signal Processing, vol. 60, pp. 866-886, 2015.

[31] Z. Chen, H. Zou, H. Jiang, Q. Zhu, Y. C. Soh, and L. Xie, "Fusion of WiFi, smartphone sensors and landmarks using the kalman filter for indoor localization," Sensors, vol. 15, no. 1, pp. 715-732, 2015.

[32] B. Allotta, L. Chisci, R. Costanzi et al., "A comparison between EKF-based and UKF-based navigation algorithms for AUVs localization," in Proceedings of the MTS/IEEE OCEANS 2015 Genova, Italy, May 2015.

[33] Y. Li, H. R. Karimi, Q. Zhang, D. Zhao, and Y. Li, "Fault Detection for Linear Discrete Time-Varying Systems Subject to Random Sensor Delay: A Riccati Equation Approach," IEEE Transactions on Circuits and Systems I: Regular Papers, 2017.

[34] Y. Li, H. R. Karimi, C. K. Ahn, Y. Xu, and D. Zhao, "Optimal residual generation for fault detection in linear discrete timevarying systems with uncertain observations," Journal of The Franklin Institute, vol. 355, no. 7, pp. 3330-3353, 2018.

[35] S. Zhao, Y. S. Shmaliy, B. Huang, and F. Liu, "Minimum variance unbiased FIR filter for discrete time-variant systems," Automatica, vol. 53, pp. 355-361, 2015. 


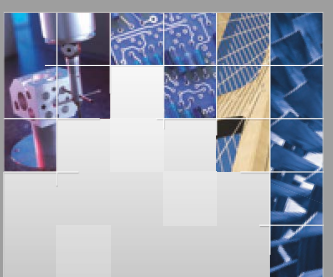

\section{Enfincering}
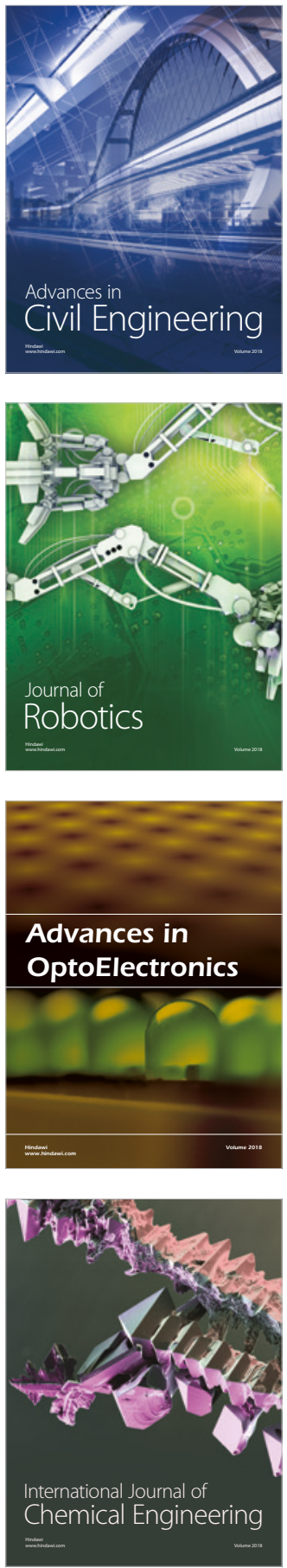

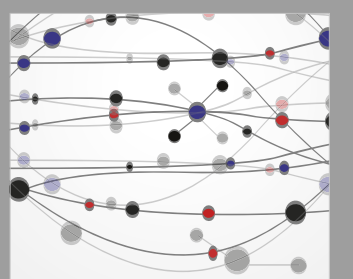

\section{Rotating \\ Machinery}

The Scientific World Journal

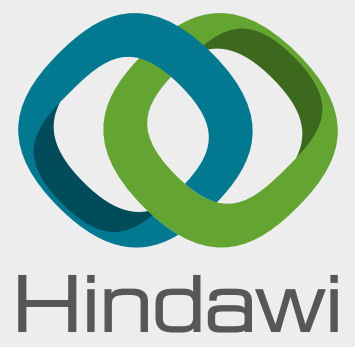

Submit your manuscripts at

www.hindawi.com
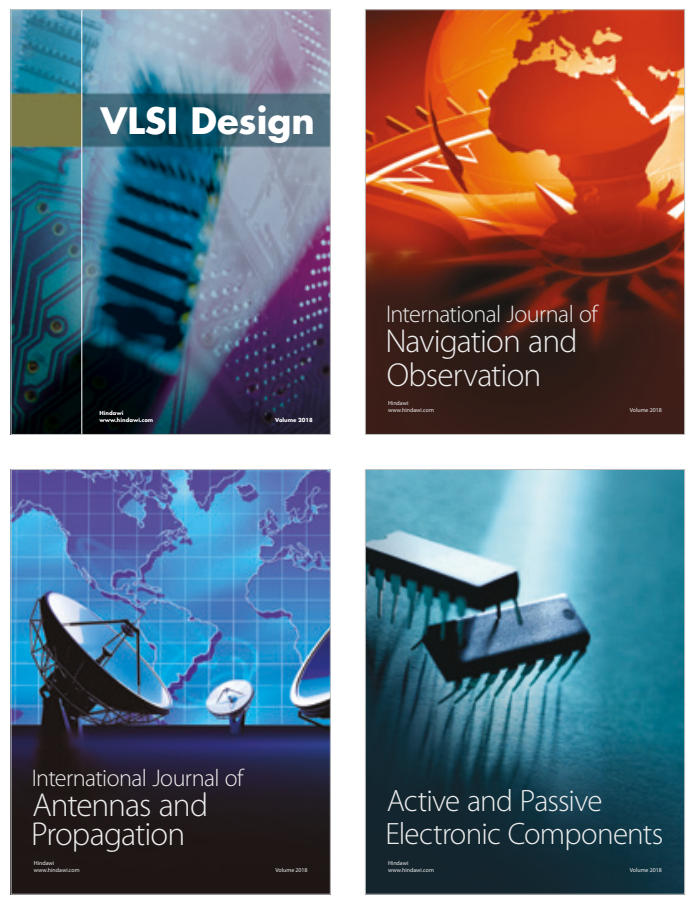
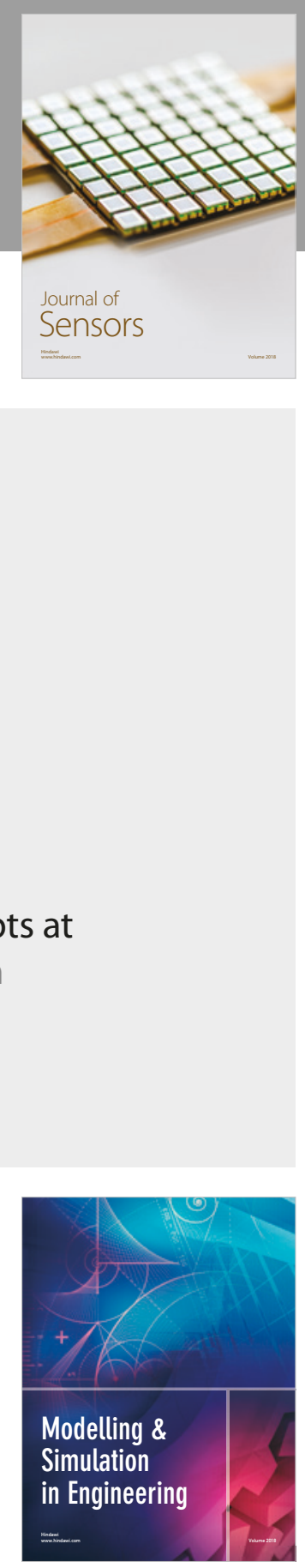

\section{Advances \\ Multimedia}
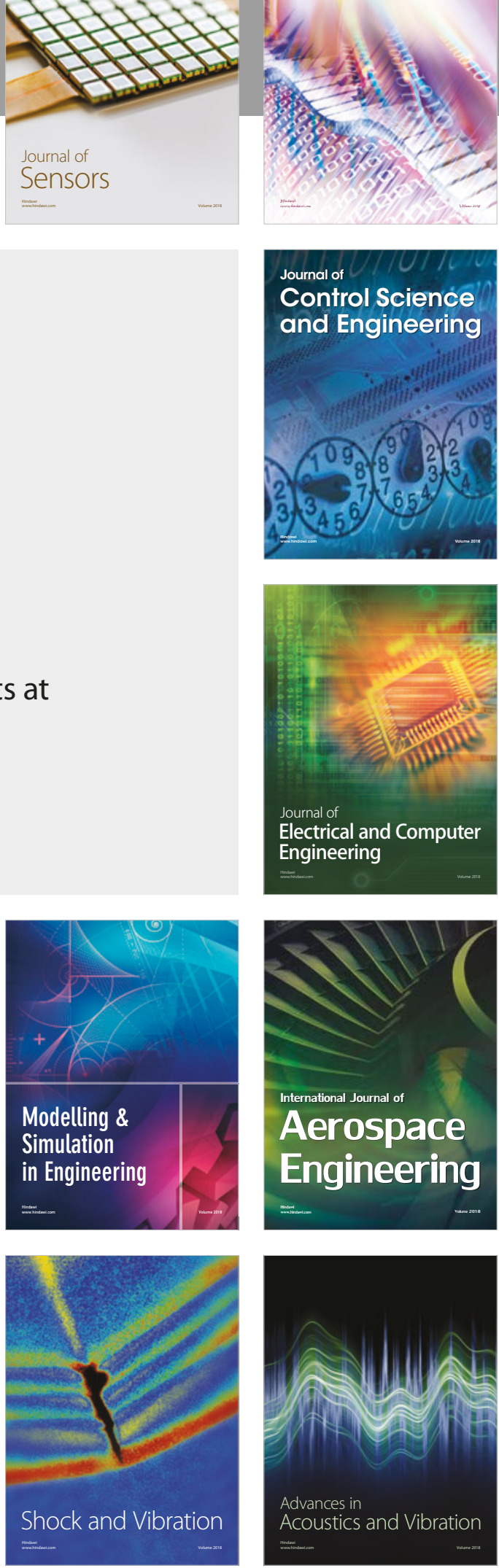